
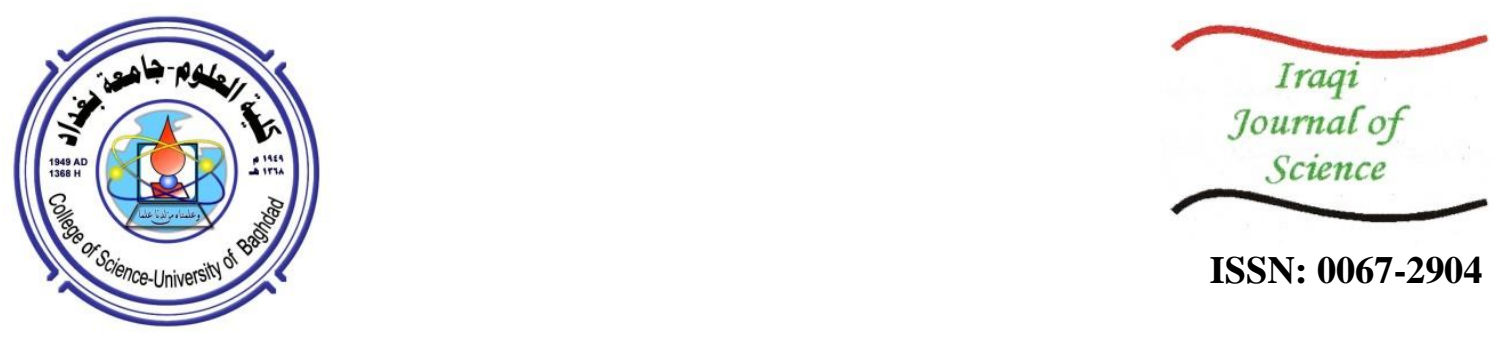

ISSN: 0067-2904

\title{
Essentially Second Modules
}

\author{
Inaam Mohammed Ali Hadi*1, Farhan Dakhil Shyaa ${ }^{2}$, Shukur Neamah Al-aeashi ${ }^{3}$ \\ ${ }^{1}$ Department of Mathematics, College of Education for Pure Sciences (Ibn-Al-Haitham) University of Baghdad, \\ Baghdad, Iraq \\ ${ }^{2}$ Department of Mathematics, College of Education, University of Al-Qadsiyah, Al-Qadsiyah, Iraq \\ ${ }^{3}$ Department of Urban Planning, College of Physical Planning, University Of Kufa, Iraq
}

\begin{abstract}
In this paper, as generalization of second modules we introduce type of modules namely (essentially second modules). A comprehensive study of this class of modules is given, also many results concerned with this type and other related modules presented.
\end{abstract}

Keywords: second modules, prime module and essentially second modules. Mathematics Subject Classification 2010:16D10, 61N60, 16D60, 16P60

\section{المقاسات الثانوبة الواسعة}
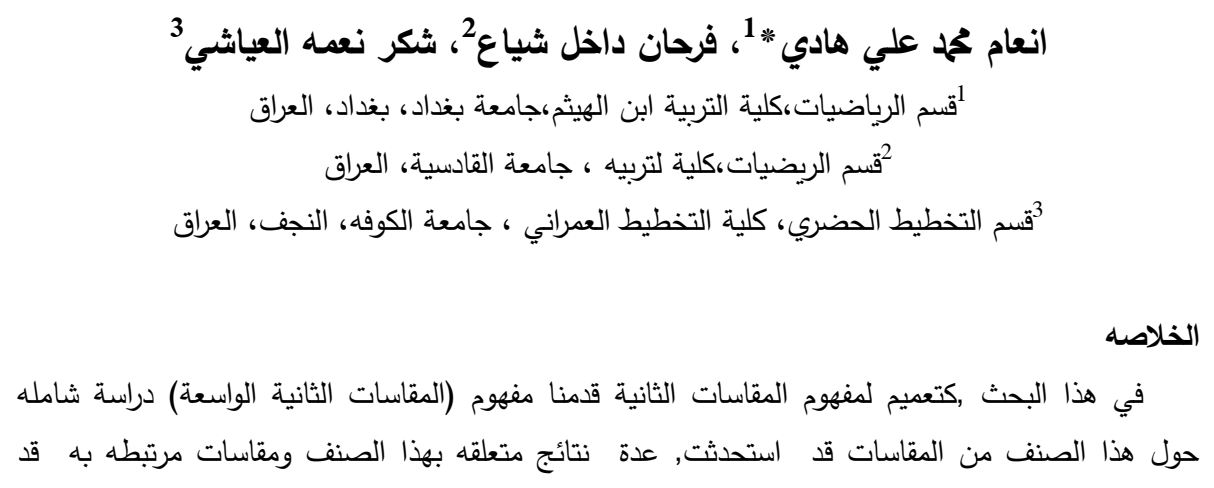

\section{Introduction}

In this research all rings are associative with identity and all modules are unitary right modules. For a right $R$-module $M$ we write $M_{R}$.Agayev in [1] defined and studied r-semisimple modules, "where an $R$-module $M_{R}$ is said to be r-semisimple if for any right ideal $I$ of $R, M I$ is a Direct summand of $M$ (briefly $M I \leq^{\oplus} M$ ) "The class of . $r$-semisimple modules contains the class of semisimple modules, also contains the class of second modules, where an $R$-module $M$ is named second if $M \neq 0$ and for each $r \in R$, either $M r=0$ or $M r=M$ [2]. Equivalently $M$ is second if for each ideal $I$ of $R$, either $M I=0$ or $M I=M[2]$. Annine in [3], [4] introduced the class of coprime modules. " An $R$-module $M$ is coprime if $\operatorname{ann}_{R}(M)=\operatorname{ann}_{R}\left(\frac{M}{N}\right)$ for each proper submodule $N$ of $M(N<M)$, where $\operatorname{ann}_{R}(M)=\{r \in R: M r=0\}^{\prime \prime}$. Wijayanti in [5] called an $R$-module $M$ is coprime if $\operatorname{ann}_{R}(M)=$ $\operatorname{ann}_{R}\left(\frac{M}{N}\right)$ for each fully invariant submodule $N$ of $M$, "where a submodule $N$ of $M$ is called fully invariant if for each endomorphism $f(f \in \operatorname{End}(M)), f(N) \subseteq N)$ " [6]. However, coprime module (in sense of Annine), coprime modules (in sense of Wijayanti) and second modules are coinciding. 
In this paper, we give another generalization of second modules. An $R$-module $M$ is an essentially second (shortly ess. second) if for each ideal $I$ of $R$, either $M I=0$ or $M I \leq_{e s s} M$. where a submodule $N$ of $M$ is essential (briefly $N \leq_{e s s} M$ ) if whenever $N \cap W=(0), W \leq M$, then $W=(0)$ [7]. Equivalently $N \leq_{e s s} M$ if and only if for each $m \in M, \exists r \in R ; \quad 0 \neq m r \in N$ [7].

It is clear that every second and uniform modules are ess. second but the converses are not true, see Remarks 2.2(2),(3).

In section two, we give the basic properties of ess. second modules such as in the class of multiplication modules, ess. second modules and uniform modules are equivalent (see, Corollary 2.4) . Every pure submodule (hence every direct summand) of ess. second modules is an ess. second module (Proposition 2.12), but the direct sum of ess. second modules may be not ess. second (see Remark 2.8). Also, if $M$ is an ess. second and $N$ is a closed submodule, then $\frac{M}{N}$ is an ess. second module (see Proposition 2.9).

In section three we present many relationships between ess. second modules and other related concept such as prime modules, r-semisimple modules (see Proposition 3.1, Theorem 3.2 and Proposition 3.3).

\section{Essentially second modules}

if $\mathrm{M}$ is an $R$-module, "a submodule $N$ of $M$ is second submodule if for each ideal $I$ of $R$, either $N I=(0)$ or $N I=N[2]$. A module $M_{R}$ is second if it is a second submodule of . A ring $R$ is a second if $R$ is a second $R$-module".

We define:

Definition 2.1: An $R$-module $M$ is called essentially second (briefly ess. second) if for each ideal $I$ of $R$, ether $M I=(0)$ or $M I \leq_{e s s} M$. A ring $R$ is ess. second if $R$ is ess. second $R$-module.

\section{Remarks 2.2:}

1- Obviously each second module is ess. second, but not conversely, as one can see by: The $Z$-module $Z_{4}$ is clearly an ess. second . and it is not second , for if $I=2 Z$, then $Z_{4}(2 Z)=<\overline{2}>\neq<$ $\overline{0}>$ and $Z_{4}(2 Z) \neq Z_{4}$.

2- Every uniform module is ess. second, but not conversely as: The $Z$-module $\mathrm{M}=Z \oplus Z$ is ess. second since for each ideal $I \neq(0)$ of $Z, I=n Z, n \in Z_{+}$, so $M I=(Z \oplus Z) n Z=n Z \oplus n Z \leq_{e s s} M$. If $I=(0)$, then $M I=(0)$. Thus $M$ is an ess. second, but is it clear that $M$ is not uniform.

3- If $R$ is an ess. second ring, then $R$ is uniform.

Let $I$ be a non-zero ideal of $R . R I=(0)$ or $R I \leq_{e s s} R$. But $R I=I$, hence $I \leq_{e s s} R$.

4- Let $M, M^{\prime}$ be $R$ - modules such that $M \simeq M^{\prime}$, then $M$ is ess. second if and only if $M^{\prime}$ is ess. second.

5- Let $A$ be an ideal of $R$ and $M$ be an $R$-module such that $M A=(0)$. Where $M$ is ess. second module $R$ - if and only if $M$ is $\frac{R}{A}$-ess. second module.

Proof: Let $0 \neq r+A \in \frac{R}{A}$. Then $r \in R, r \neq 0$. Since $M$ is an ess. second $R$-module, either $M r=0$ or $M r \leq_{e s s} M$. If $M r=0$, then $r \in A$ and $m(r+A)=0$.If $M r \leq_{e s s} M$, then $M(r+A)=M r \leq_{e s s} M$. Thus $M$ is an ess. second $\frac{R}{A}$-module. The proof of converse is similarly.

6- r-semisimple module and ess. second module are independent concepts. For examples The $Z$ module $Z_{6}$ is r-semisimple but it is not ess. second. While The $Z$-module $Z_{4}$ is an ess. second module, but it is not r-semisimple. Also, it is not second.

7- Let $M$ be a torsion free $R$-module and $R$ is ess. second. Then for each $m \in M, m R$ is an ess. second.

The pursue is a characterization of ess. second modules.

Theorem 2.3: For an $R$-module $M_{R}$, the following statements are equivalent:

1- $\quad M$ is ess. second;

2- $\quad$ If $0 \neq N \leq M, N=M[N: M]$, then $N \leq_{\text {ess }} M$;

3- For each $r \in R$, either $M r=0$ or $M r \leq_{e s s} M$.

Proof: (2) $\Rightarrow(1)$ Let $I$ be an ideal of R. Assume $M I \neq 0$. Set $N=M I$. It is clear that $M I=M[M I: M]$; that is $N=M[N: M]$ and so by (2) $N=M I \leq_{\text {ess }} M$.

$(1) \Rightarrow(3)$ It is obvious.

(3) $\Rightarrow(2)$ Let $0 \neq N=M[N, M]$. Then there exists $r \in[N: M]$ such that $M r \neq(0)$, so that $M r \leq_{\text {ess }} M$ by condition (3). But $M r \leq M[N: M]=N$. This implies $N \leq_{\text {ess }} M$. 
it is known that: an R-module $\mathrm{M}$ is called a multiplication module provided for each submodule $\mathrm{N}$ of $\mathrm{M}$ there exists an ideal I of $\mathrm{R}$ such that $\mathrm{N}=\mathrm{MI}$. [8]

Corollary 2.4: For a multiplication module $M$ over a ring $R$. The pursue are equivalent:

$1-M$ is ess. second;

2- $M$ is uniform;

3- For each $r \in R$ either $M r=0$ or $M r \leq_{\text {ess }} M$.

Note that the condition $M$ is multiplication can't be dropped from Corollary 2.4, since the Zmodule $Q \oplus Q$ is ess. second and it is not uniform.

Corollary 2.5: Let $R$ be a commutative ring Then $R$ is ess. second if and only if $R$ is uniform.

Corollary 2.6: For a faithful multiplication module over a ring $R$. The pursue are equivalent:

1- $M$ is an ess. Second ;

2- $M$ is uniform ;

3- $R$ is uniform ;

4- $R$ is ess.second.

Proposition 2.7: Let $\mathrm{M}$ be an ess. second module and let $N \leq{ }^{\oplus} M$.Then $\mathrm{N}$ is ess. second.

Proof: Let $M_{1} \leq^{\oplus} M$. Then $M=M_{1} \oplus M_{2}$ for some $M_{2} \leq M$.For any ideal $I$ of $R$, either $M_{1} I=0$ or $M_{1} I \neq 0$. If $M_{1} I \neq 0$ then $M I=M_{1} I \oplus M_{2} I \neq 0$. Hence $M I \leq_{e s s} M$ and this implies $M_{1} I \leq_{e s s} M_{1}$, by [7, Prop. 1.1,P.16] Thus $M_{1}$ is an ess. second module.

Remark 2.8: The direct sum of ess. second modules is not necessary ess. second, for example:

Each of the $Z$-module $Z_{3}$ and $Z_{4}$ is an ess. second module but $Z_{3} \oplus Z_{4} \simeq Z_{12}$ is not an ess. second module since $Z_{12}(3 Z)=<\overline{3}>\$_{\text {ess }} Z_{12}$ and $Z_{12}(3 Z) \neq<\overline{0}>$.

Proposition 2.9: For any ess. second module $M, \oplus_{i \in I} M_{i}\left(M_{i}=M\right.$, for each $\left.i \in I\right)$ is an ess. second.

Proof: It is easy.

A submodule $N$ of an $R$-module $M$ is closed if $N$ has no proper essential extension, [7].

Proposition 2.10: Let $N$ be a closed submodule of an ess. second module $M$. Then $\frac{M}{N}$ is an ess. second module.

Proof: Let $I$ be an ideal of $R$. Since $M$ is an ess. second module, either $M I=(0)$ or $M I \leq_{e s s} M$. If $M I=(0)$, then $\frac{M}{N} I=\frac{M I+N}{N}=\left(0_{\frac{M}{N}}\right)$. If $M I \leq_{e s s} M$, then $M I+N \leq_{e s s} M$, and since $N$ is closed in $M$, then $\frac{M I+N}{N} \leq_{e s s} \frac{M}{N}$ by [7, Proposition $\left.1.4(\mathrm{a} \Leftrightarrow \mathrm{b})\right]$. It follows that $\frac{M}{N} I \leq_{e s s} \frac{M}{N}$. Thus $\frac{M}{N}$ is an ess. second.

Remark 2.11: The condition ( $N$ is closed in $M$ ) is a necessary condition in Proposition 2.10, for example. The $Z$-module $Z$ is an ess. second (since it is second). But $\frac{Z}{12 Z} \simeq Z_{12}$ is not ess. second and $12 Z$ is not closed in $Z$.

Corollary 2.12: Let $f: M \mapsto M^{\prime}$ be an epimorophism such that $\operatorname{Ker}(f)$ is closed and $M$ is an ess. second. Then $M^{\prime}$ is an ess. second.

By applying Proposition 2.10 we can give a different proof of Proposition 2.7 as follows

Proof: Since $N \leq^{\oplus} M$, then $N \oplus W=M$ for some $W \leq M$. But $W \leq^{\oplus} M$, implies $W$ is closed submodule of $M$ [7,Exc.3,P.19] Hence $\frac{M}{W}$ is an ess. second by Proposition 2.10 and this implies $N$ is an ess. second since $N \simeq \frac{M}{W}$.

A submodule $N$ of an $R$-module $M$ is called pure if $M I \cap \mathrm{N}=\mathrm{NI}$ for each ideal $I$ of $R$,[9]

Proposition 2.13: Every pure submodule of ess. second module is an ess. second.

Proof: Let $N$ be a pure submodule of $M$, let $I$ be an ideal of $R$. Since $M$ is an ess. second either $M I=(0)$, or $M I \leq_{e s s} M$. If $M I=(0)$,then $N I=(0)($ since $N \leq M)$, if $M I \leq_{e s s} M$, then $M I \cap$ $\mathrm{N} \leq_{e s s} M \cap \mathrm{N}=\mathrm{N}$ and so $N I \leq_{e s s} N$. Thus $N$ is an ess. second.

Since every direct summand of a module is pure, we can also get Proposition 2.7 directly, by Proposition 2.13.

Proposition 2.14: Let $M$ be an $R$-module. $M$ is an ess.second as a left $E$-module if and only if for each $0 \neq f \in \operatorname{Hom}(M, N), N \leq M$ implies $N \leq_{e s s} M$. Where $E=\operatorname{End}(M)$.

Proof: $\Rightarrow$ Let $0 \neq f \in \operatorname{Hom}(M, N)$. Then $i \circ f \in E$, where $i$ is the inclusion mapping from $N$ to $M$. Since $M$ is an ess. second $E$-module, either $(i \circ f)(M)=(0)$ or $(i \circ f)(M) \leq_{\text {ess }} M$. But $(i \circ$ $f)(M)=(0)$ implies $f=0$ which is a contradiction, hence $(i \circ f)(M) \leq_{e s s} M$; that is $f(M) \leq_{\text {ess }} M$. But $f(M) \leq N$, so that $N \leq_{\text {ess }} M$. 
$\Leftarrow$ To prove $M$ is an ess. second $E$-module. That is to prove for each $f \in E$, either $f(M)=(0)$ or $f(M) \leq_{e s s} M$. suppose that $f(M) \neq 0$ that is $f \neq 0$. Put $N=f(M)$, hence $f \in \operatorname{Hom}(M, N)$ and by hypothesis $N \leq_{e s s} M$. Thus $f(M) \leq_{e s s} M$.

\section{Essential Second Modules and other related concept}

In this section many connections between ess. second modules and other related concepts are presented.

First we have

Proposition 3.1: An $R$-module $M$ is an ess. second and r-semisimple iff $M$ is second.

Proof: $\Rightarrow$ Let $I$ be an ideal of $R$. If $M I=(0)$, then nothing to prove. If $M I \neq(0)$, then $M I \leq_{e s s} M$, since $M$ is ess. second. But $M$ is an r-semisimple, so that $M I \leq^{\oplus} M$. It follows that $M I=M$. Thus $M$ is second.

$\Leftarrow$ It is obvious.

An $R$-module $M$ is prime if $\operatorname{ann}(M)=\operatorname{ann}(N)$ for each $(0) \neq N \leq M$ [10]. A proper submodule $N$ of an $R$-module is prime if whenever $x \in M, r \in R, x r \in N$ implies $x \in N$ or $r \in$ [N:M] [10]. $M$ is a prime. if and only if (0) is a prime submodule of $M^{\prime \prime}$.

Theorem 3.2: Let $\mathrm{M}$ be a prime. over a commutative ring. $\mathrm{R}$ and let $\mathrm{N}<M$ such that $N$ is an ess. second submodule. Then $N$ is a prime submodule.

Proof: Let $x \in M, r \in R$ with $x r \in N$. Suppose $x \notin N$, so we must prove $r \in[N: M]$. Since $N$ is an ess. second, either $N r=(0)$ or $N r \leq_{\text {ess }} N$. If $N r=(0)$, then $r \in \operatorname{ann}(N)=\operatorname{ann}(M)$ and this implies $r \in[N: M]$. If $N r \leq_{e s s} N$, then there exists $a \in R$ such that $0 \neq x r a \in N r$. Thus $x r a=n r$ for some $n \in N$. Since $R$ is commutative, $x r a=x a r$, hence $x a r=n r$ which implies $(x a-n) r=0$; that is $r \in \operatorname{ann}(x a-n)$. But $\operatorname{ann}(x a-n)=\operatorname{ann}(M)$ (since $M$ is prime.). Therefore $r \in \operatorname{ann}(M) \subseteq$ $[N: M]$. Thus $N$ is a prime submodule.

Proposition 3.3: Let $M$ be a prime $R$-module, $N=x R$ for some $x \in M$. If $N$ is an ess. second $R$ module, then $M$ is an ess. second.

Proof: Let $r \in R$. Suppose $M r \neq(0)(r \notin \operatorname{ann}(M))$. Hence $r \notin \operatorname{ann}(N)$ (since $M$ is a prime.). So $N r \neq(0)$, but $N$ is an ess. second module implies $N r \leq_{\text {ess }} N=x R$. Now $x \in N$, hence there exists $r^{\prime} \in R$ such that $0 \neq x r^{\prime} \in N r$. It follows that $x r^{\prime}=x a r$ for some $a \in R$. Thus $x\left(r^{\prime}-a r\right)=0$; that is $r^{\prime}-\operatorname{ar} \in \operatorname{ann}(X)=\operatorname{ann}(M)$. Hence for each $m \in M, m r^{\prime}=\operatorname{mar}$ and $0 \neq m r^{\prime}$ (because if $m r^{\prime}=0$ then $r \in \operatorname{ann}(m)=\operatorname{ann}(M)$ and so $M r=0$ which is a contradiction). Therefore, $\forall m \in M$ , there exists $r^{\prime} \in R$ such that $0 \neq m r^{\prime}=\operatorname{mar} \in M r$. Thus $M r \leq_{e s s} M$ and $M$ is an ess. second module.

Proposition 3.4: Let $N \leq_{e s s} M$, $\operatorname{ann}(M)=\operatorname{ann}(N)$. If $N$ is an ess. second submodule of $M$. Then $M$ is an ess. second module.

Proof: Let $r \in R$. Since $N$ is an ess.second submodule, then either $N r=(0)$ or $N r \leq_{e s s} N$. If $N r=(0)$, then $M r=(0)\left(\right.$ since $\operatorname{ann}(M)=\operatorname{ann}(N)$ by hypothesis). If $N r \leq_{e s s} N$, then $N r \leq_{e s s} M$ since $N \leq_{e s s} M$. But $M r \supseteq N r$, hence $M r \leq_{e s s} M$. Thus $M$ is ess. second.

Remark 3.5: The condition $\operatorname{ann}(M)=\operatorname{ann}(N)$ is necessary condition, for example. Let $M$ be the $Z$ module $M=Z_{2} \oplus Z_{4}$. Let $N=Z_{2} \oplus<\overline{2}>\leq_{\text {ess }} M$, $\operatorname{ann}(M)=4 Z \neq \operatorname{ann}(N)=2 Z$. But $N \simeq Z_{2} \oplus Z_{2}$ so that $N$ is an ess. second. But $M$ is not an ess. second module since $M(2 Z)=(\overline{0}) \oplus \quad<\overline{2}>\neq 0$ and $M(2 Z) \$_{\text {ess }} M$.

An $R$-module $\mathrm{M}$ is called coquasi-Dedekind if $\operatorname{Hom}(M, N)=(0)$ for each $N \lesseqgtr M$ [11]. Equivalently $M$ is coquasi-Dedekind if for each $0 \neq f \in \operatorname{End}(M), f$ is an epimorophrism".

We present the following

Definition 3.6: An $R$-module $M$ is to be essentially coquasi-Dedekind if for each $f \in \operatorname{End}(M) . \operatorname{Imf} \leq_{\text {ess }} M$.

Note that Sahra in [11] gave the following: an $R$-module $M$ is called essentially coquasi-Dedekind if for each $(0) \neq f \in \operatorname{End}(M), \operatorname{Ker}(f) \leq_{\text {ess }} M$. However our definition is different of that was given in [11]. 


\section{Examples 3.7:}

1- Every simple module (and the $Z$-modules $Z, Q$ )are ess. coquasi-Dedekind in sense of Definition 3.6, but it is not ess. coquasi-Dedekind in sense of [11].

2- Consider $\boldsymbol{Z}_{\mathbf{1 2}}$ as $Z$-modules, is an ess. couasi-Dedekind in sense of [11]. But it is not ess. coquasiDedekind in sense of Definition 3.6, since there exists $f: Z_{12} \mapsto Z_{12}$ define by $f(x)=6 x$ for each $x \in Z_{12}$ and $\operatorname{Imf}=<6>\$_{\text {ess }} Z_{12}$

Remark 3.8: Every ess. coquasi-Dedekind module is ess. second.

Proof: Let $r \in R$. If $M r \neq(0)$. Define $f: M \mapsto M$ by $f(m)=m r$ for each $m \in M, 0 \neq f$. Then $\operatorname{Im} f=M r$. But $\operatorname{Im}(f) \leq_{\text {ess }} M$ since $M$ is ess. coquasi-Dedekind. Thus $M r \leq_{e s s} M$.

Note that the reverse is not achievable in public as: let $M=Q \oplus Q$ as $Z$-module. $M$ is ess. second module, but it is not ess. coquasi- Dedekind since $\exists f \in \operatorname{End}(M)$ such that $f(x, y)=(x, 0)$, for each $(x, y) \in M$ and so $\operatorname{Im}(f)=Q \oplus(0) \$_{\text {ess }} M$.

An $R$-module $M$ is scalar module if for each $f \in \operatorname{End}(M), \exists 0 \neq r \in R, f(m)=m r, \forall m \in M$ [12].

Proposition 3.9: Let $M$ be a scalar module. Then $M$ is an ess. coquasi-Dedekind iff $M$ is an ess. second module.

Proof: It is easy, so is omitted.

The following result follows directly.

Proposition 3.10: Let $M$ be an $R$-module. Then $M$ is an ess. coquasi-Dedekind iff $M$ is an ess.secend left $E$-module, where $E=\operatorname{End}(M)$.

By combining Proposition 3.10 and Proposition 2.13, we have the following:

Corollary 3.11: For an $R$-module $\mathrm{M}$. The pursue are synonymous:

1- $M$ is an ess. coquasi-Dedekind $R$-module;

2- $\operatorname{Hom}(M, N) \neq 0($ where $N \leq M)$ implies $N \leq_{\text {ess }} M$;

3- $M$ is an ess. second left $E$-module.

As we mention in the introduction the second module is called coprime by some authors, see[2,13]. Sahera in [11] introduced the concept ess. coprime as a generalization of coprime ( second module) where an $R$-module is referred by an ess. coprime if for each $r \in R$, either $M r=M$ or $\operatorname{ann}_{M}(r) \leq_{e s s} M$, where $a n n_{M}(r)=\{m \in M: m r=0\}$.

Notice that the concept ess. second is independent with ess.coprime[11]. Like:

1- Let $M=Z_{2} \oplus Z$ as $Z$-module. It is easy to see that $M$ is an ess. coprime and it is not ess. second.

2- For the $Z$-module $M=Z \oplus Z$. $M$ is ess. second. But for any $0 \neq r \in Z$, $\operatorname{ann}(r)=\{(a, b) \in$ $M:(a, b) r=(0,0)\}=(0) \Varangle_{e s s} M$. Also, $M r \neq M$ for each $r \in Z, r \neq \pm 1$. Thus $M$ is not ess. coprime.

It is known that for every second $R$-module $\quad \operatorname{ann}_{Z}(M)$ a prime ideal. of $R$. However this is not true for ess. second module as we have:- the $Z$-module $Z_{8}$ is an ess. Second (since it is uniform ) and $\operatorname{ann}_{Z}\left(Z_{8}\right)=8 Z$ which is not a prime ideal. of $Z$.

In [13] we define the concept essential prime (briefly ess . prime ) as follows : an $R$-module $\mathrm{M}$ is said to be an ess. prime whenever $\operatorname{ann}_{R}(M)=\operatorname{ann}_{R}(N)$ for all $N \leq_{\text {ess }} M$."

We state and prove the pursue :

Proposition 3.12: Let $M$ be an ess. second $R$-module and ess. prime.. Then $a n n_{R}(M)$ is a prime ideal. of $R$.

Proof: Let $a, b \in R$ and $a . b \in \operatorname{ann}_{R}(M)(M a b=0)$. Assume $a \notin a n n(M)$, that is $M a \neq(0)$. Since $M$ is ess. second, then $M a \leq_{\text {ess }} M$. on the other hand $M$ is ess. prime, so $a n n_{R}(M)=a n n_{R}(M a)$. But $b \in \operatorname{ann}_{R}(M)$ ( since $M a b=(0)$ ) hence $b \in a n n_{R}(M)$. Thus $a n n_{R}(M)$ is a prime ideal.

Note that ess. the second module does not imply ess. prime., as the $Z$-module $M=Z_{4}$ is ess. second, however it is not ess. prime since $\operatorname{ann}_{Z}(M)=4 Z \neq a n n_{Z}(\overline{2})=2 Z$, and $(\overline{2}) \leq_{\text {ess }} Z_{4}$. Also, ess. prime. does not imply ess. second, as: The $Z$-module $M=Z_{2} \oplus Z$ is an ess. prime and it is not an ess. second.

Corollary 3.13: Let $M$ be an $R$-module and every prime ideal. of $R$ is maximal. Then the pursue are synonymous:

1- $M$ is second;

2- $M$ is prime.;

3- $M$ is an ess. prime. and ess. second;

4- $\operatorname{ann}_{R}(M)$ is a prime ideal of $R$. 
Proof: $(1) \Leftrightarrow(2)$. [14, Lemma 1.1]

(2) $\Rightarrow$ (4) It is clear.

(4) $\Rightarrow$ (2) $\operatorname{ann}_{R}(M) \subseteq \operatorname{ann}_{R}(N)$ for each $0 \neq N \leq M$. But $a n n_{R}(M)$ is a prime ideal. by condition

(4), so $\operatorname{ann}_{R}(M)$ is maximal and so $a n n_{R}(M)=\operatorname{ann}_{R}(N)$. Thus $M$ is a prime module..

(3) $\Rightarrow(2)$ By Proposition 3.12, $\operatorname{ann}_{R}(M)$ is a primeideal., hence $\operatorname{ann}_{R}(M)$ is maximal by hypothesis.

But $\operatorname{ann}_{R}(M) \subseteq \operatorname{ann}_{R}(N)$ for each $0 \neq N \leq M$ so that $\operatorname{ann}_{R}(M)=\operatorname{ann}_{R}(N)$. Thus $M$ is prime..

(2) $\Rightarrow$ (3) Since $M$ is prime., then $M$ is an ess. prime. But $M$ is prime implies $M$ is second by (part (2) $\Leftrightarrow(1))$, hence $M$ is ess. second.

It is known that if $R$ is an Artinian ring or a Boolean ring, then every prime ideal. is maximal. Hence we get.

Corollary 3.14: Let $M$ be an $R$-module where $R$ is an Artinian ring or Boolean ring. Then the pursue is synonymous.

1- $M$ is second ;

2- $M$ is prime ;

3- $M$ is ess. prime and ess. second;

4- $\operatorname{ann}_{R}(M)$ is a prime ideal. Of $R$.

Proposition 3.15: Let $M$ be an $R$-module such that $\operatorname{ann}_{R}(M)$ is semisimple and $\operatorname{ann}_{R}(N)=$ $\operatorname{ann}_{R}\left(\frac{M}{N}\right)$, for each $N \lesseqgtr M$. Then $M$ is prime and second module.

Proof: To prove $M$ is prime. Let $r \in \operatorname{ann}_{R}(N)$. Then $N r=0$ and so $\frac{M}{N} \mathrm{r}=0$, by hypothesis; that is $M r \subseteq N$. Thus $M r^{2} \subseteq N r=(0)$. Thus $M r^{2}=0$ which implies $M r=0(r \in \operatorname{ann}(M))$ since $\operatorname{ann}(M)$ is semi prime. Hence, $\operatorname{ann}_{R}(M)=\operatorname{ann}_{R}(N)$. Therefore $M$ is prime. But $\operatorname{ann}(N)=\operatorname{ann}\left(\frac{M}{N}\right)$ so that $\operatorname{ann}_{R}(M)=\operatorname{ann}_{R}\left(\frac{M}{N}\right)$ for each $N<M$. Hence $M$ is second.

An $R$-module $M$ is homogenous semisimple if $M$ is a direct sum of pair wise isomorphic simple submodules, [14]. In the last part of Lemma 1.1 in [14]. If $M$ is a module over a commutative $R$ such that every prime ideal . is maximal, then $M$ is second iff $M$ is a homogenous semisimple.

Corollary 3.16: If $M$ is an $R$-module, where $R$ is a commutative ring. such that every prime ideal. is maximal (hence if $R$ is Artinian ring or Boolean or Von Neumman regular). Then the pursue are synonymous:

1- $M$ is second ;

2- $M$ is prime.;

3- $M$ is an ess. prime and ess. second module;

4- $\operatorname{ann}(M)$ is a maximal ideal;

5- $M$ is a homogenous semisimple.

Proposition 3.17: Let $M$ be multiplication module over a ring $R$. Then $M$ is a second if and only if $M$ is a homogenous semisimple.

Proof: $\Rightarrow$ Since $M$ is a multiplication module then for each proper submodule $\mathrm{N}$ of $\mathrm{M}, \mathrm{N}=\mathrm{M}[\mathrm{N}: \mathrm{M}$ ].=M ann $\frac{M}{N}$. Because $\mathrm{M}$ is second, ann $\frac{M}{N}=$ ann $\mathrm{M}$, hence $\mathrm{N}=\mathrm{M}$ ann $\mathrm{M}=0$ Then $M$ is simple. Thus $M$ is homogenous semisimple.

$\Leftarrow \mathrm{It}$ is given in [14].

Corollary3.18: Let $R$ be a commutative ring. Then $R$ is second if and only if $R$ is homogenous semisimple

\section{References}

1. NAZIM AGAYEV, CES IM ÇEL'IK and TAH'IRE ÖZENProc. 2018. "On a generalization of semisimple modules" Indian Acad. Sci. (Math. Sci.), 128(20): 1-10.

2. Yassemi "The dual notion of prime submodules" Arch. Math. (Bron), 37 (2001): 273-288.

3. Annin, S. 2008. Attached primes over noncommutative rings. J. Pure Appl. Algebra, 21: 510521.

4. Annine S. 2002. "Associated and attached prime over non commutative ring" Ph.D. University of Berkeley. 
5. Wijayanti I.E. 2006. "Coprime Modules and comodules", ph.D. Thesis, Heinrich-Heine Unoversitat Dusseldrof.

6. Wisbauer, R. 1991. "Foundations of Modules and Rings theory", reading: Gordon and Breach.

7. Goodearl K.R. 1976. " Ring Theory, Non Singular Rings and Modules. Marcel Dekker", Inc. New York and Basel.

8. Zeinab, A.EL-Bast and Pattrick F.Smith, 1988. " Multiplication Modules " Communication in Algebra, 10(4): 755-779.

9. Anderson, F. W., Fuller K. R. 1992. "Rings and Categories of Modules", Second Edition, Graduate Texts in Math., Vo1.13, Springer-Verlag, Berlin-Heidelberg-New York.

10. Beachy, J. 1975. Some aspects of noncommutative localization, in Noncommutative Ring Theory, Kent State University, Lecture Notes in Mathematics, Vol. 545, Springer-Verlag, Berlin-New York.

11. ahera, M. Y. 2003. "Coquasi-Dedekind Modules" Ph.D. thesis Univ. of Baghdad.

12. Shihab B.N. 2004. "Scalar Reflexive Modules". Ph.D. Thesis, College of Education Ibn ALHaitham, University of Baghdad.

13. Enaam Mohammed Ali and Tha'ar Younis. 2011. Essentially prime Modules and Related. J. of Basrah researchers (sciences), 37(4), (2011).

14. Çeken S., Alkan M. and Smith P. F. 2013. Second Modules over Noncommutative Rings, Communications in Algebra, 41(1): 83-98. 